\title{
MELANOQUERATOSIS ESTRIADA POSTRAUMÁTICA EN MUJER DE RAZA BLANCA
}

\author{
POST-TRAUMATIC STRIATE MELANOKERATOSIS IN A \\ CAUCASIAN WOMAN
}

\author{
BOTO-DE-LOS-BUEIS A ${ }^{1}$, DÍAZ-VALLE D² ${ }^{2}$ LLORENTE-GONZÁLEZ S ${ }^{3}$, \\ PASTORA-SALVADOR N³ , PATRÓN-ROMERO M4, HERNÁNDEZ-GONZÁLEZ C ${ }^{3}$
}

\begin{abstract}
RESUMEN
Caso clínico: Se presenta el caso de una mujer de raza blanca y piel clara, con erosión corneal recurrente y con melanosis conjuntival en OI, que desarrolló una opacidad corneal por melanoqueratosis estriada. La melanosis conjuntival se trató con mitomicina tópica, manteniendo la pigmentación corneal.

Discusión: La melanoqueratosis estriada se presenta en personas de piel oscura, con un único caso descrito en personas de raza caucásica. Ésta se origina por daño corneal o por migración de pigmento desde melanosis conjuntivales, debiendo tratar ambas condiciones para detener la pigmentación corneal.
\end{abstract}

Palabras clave: Melanoqueratosis estriada, erosión corneal recurrente, pigmentación corneal, mitomicina $\mathrm{C}$ tópica, melanosis conjuntival, raza caucásica.

\section{INTRODUCCIÓN}

La melanoqueratosis estriada (ME) es una pigmentación corneal que puede acompañar al proceso

\begin{abstract}
Clinical case: A fair-skinned woman presented marked striate melanokeratosis in her left eye related to recurrent corneal erosion. The source of pigmentation was a conjunctival melanosis. The conjunctival melanosis responded to treatment with topical mitomycin, while the corneal pigmentation persisted. Discussion: Striate melanokeratosis is a condition described in dark-skinned patients who show a well-defined pigmentation of the limbal area, with only one case of striate melanokeratosis reported previously in a Caucasian person. The stimuli for this proliferation are corneal lesions or melanosis close to the limbus. Avoiding both stimuli are the main steps in its management (Arch Soc Esp Oftalmol 2009; 84: 213-216).
\end{abstract}

Key words: Striate melanokeratosis, recurrent corneal erosion, corneal pigmentation, topical mitomycin $\mathrm{C}$, conjunctival melanosis, caucasian race.

\footnotetext{
Recibido: 19/3/07. Aceptado: 27/3/09.

${ }^{1}$ Doctor en Medicina. Servicio de Oftalmología. Hospital Universitario La Paz. Madrid. España.

2 Doctor en Medicina. Servicio de Oftalmología. Hospital Universitario Clínico. Madrid. España.

${ }^{3}$ Licenciado en Medicina. Servicio de Oftalmología. Hospital Universitario La Paz. Madrid. España.

${ }^{4}$ Licenciado en Medicina. Servicio de Anatomía Patológica. Hospital Universitario La Paz. Madrid. España.

Correspondencia:

Ana Boto de los Bueis

Servicio de Oftalmología. Hospital Universitario La Paz

Paseo de la Castellana, 261

28046 Madrid

España

E-mail: botobueis@ telefonica.net
}

de curación de una erosión epitelial corneal en personas de raza oscura. Presentamos a una paciente de raza blanca que desarrolló una $\mathrm{ME}$ en relación a erosión corneal recurrente en su ojo izquierdo (OI). 


\section{CASO CLÍNICO}

Mujer de 50 años con antecedentes de erosiones corneales en OI desde hace 6 años, de origen desconocido. En noviembre de 2004 acude por dolor OI destacando una agudeza visual (AV) de 1 y una erosión temporal-superior próxima a limbo. La conjuntiva bulbar de OI presenta dos parches de melanosis conjuntival en cuadrantes temporal (fig. 1) e inferior. La córnea muestra pigmentación subepitelial difusa, más acentuada en periferia y limbo superior y temporal, sin vascularización corneal (fig. 2). La erosión es tratada con desepitelización, lente de contacto terapéutica (LCT), y colirios de ácido hialurónico, suero autólogo $20 \%$, antiedema y fluorometalona, esta última durante 2 semanas, realizándose posteriormente micropunciones por recurrencia de la erosión en esta zona. En mayo de 2005 se añade al tratamiento doxiciclina $100 \mathrm{mg} / 24 \mathrm{~h}$ y vitamina C $1 \mathrm{gr} / 24 \mathrm{~h}$ por erosión nasal-superior periférica. En julio de 2005 presenta una erosión temporal. En diciembre de 2005 presenta erosión corneal nasal superior muy dolorosa realizándose desepitelización amplia; en la base de la úlcera se observa pigmento. El epitelio corneal se remite a anatomía patológica mostrando células melánicas HMB-45 positivas características de melanocitos. La reepitelización de esta zona se acompaña de una intensa pigmentación local. En febrero de 2006 se realiza biopsia conjuntivo-limbar temporal inferior que muestra mucosa conjuntival con metaplasia

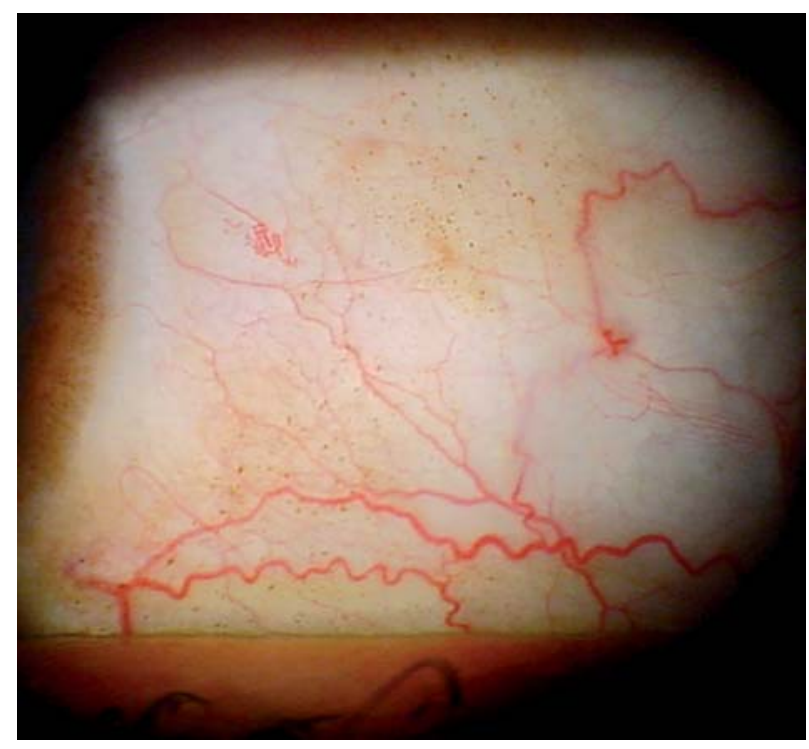

Fig. 1: Melanosis conjuntival temporal OI.

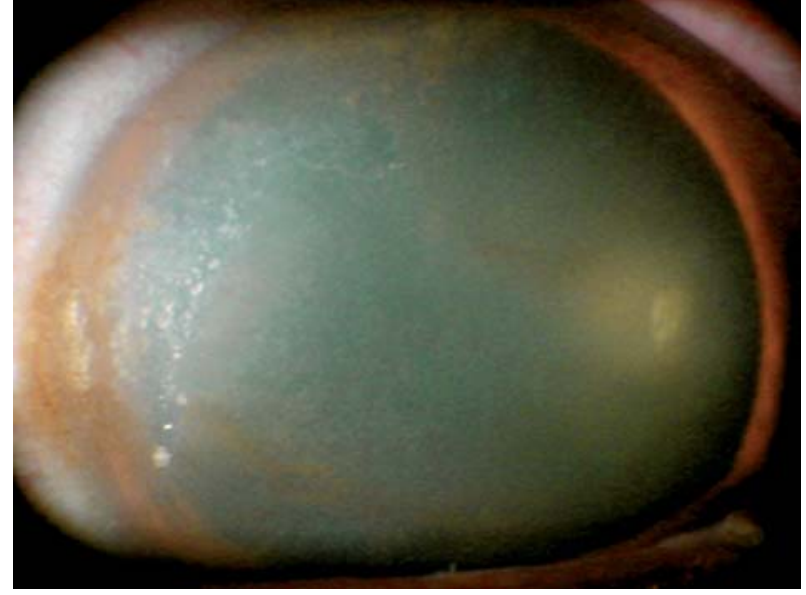

Fig. 2: Pigmentación limbar y corneal difusa.

escamosa e hiperpigmentación melanocítica basal, sin melanocitos anormales y biopsia corneal del mismo cuadrante en la que se describe hiperpigmentación melanocítica basal por depósito de melanocitos (fig. 3). Durante la evolución la paciente mantiene molestias continuas OI y progresión de la pigmentación corneal con leve descenso de AV OI a 1 (-3). En marzo de 2006 se inicia tratamiento con colirio de mitomicina $\mathrm{C}$ al $0,02 \% / 8 \mathrm{~h}$ y colirio de fluorometalona/ $8 \mathrm{~h}$ durante dos semanas, repitiendo este ciclo una semana más con un intervalo de 3 semanas. Biomicroscópicamente desaparece la pigmentación conjuntival, pero persiste la melanosis corneal acompañándose en el 2008 de recurrencia de erosión corneal supero-temporal y súpero-nasal (fig. 4).

\section{DISCUSIÓN}

Alrededor del limbo corneal existe una banda de pigmento cuya densidad varía con la raza. La mayo-

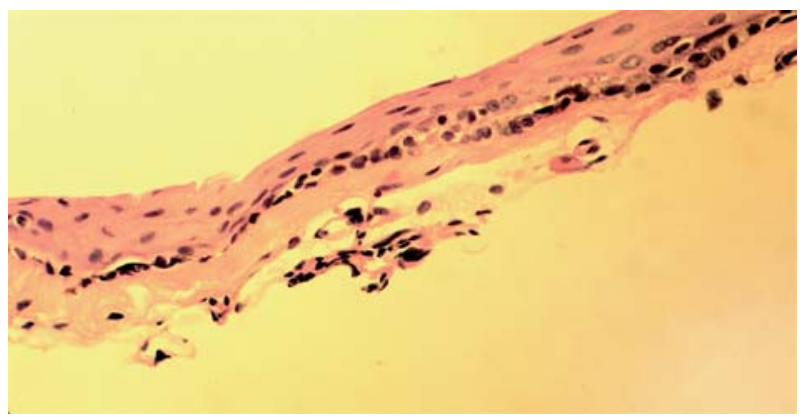

Fig. 3: Biopsia corneal con depósito de melanocitos en las capas basales del epitelio corneal. 


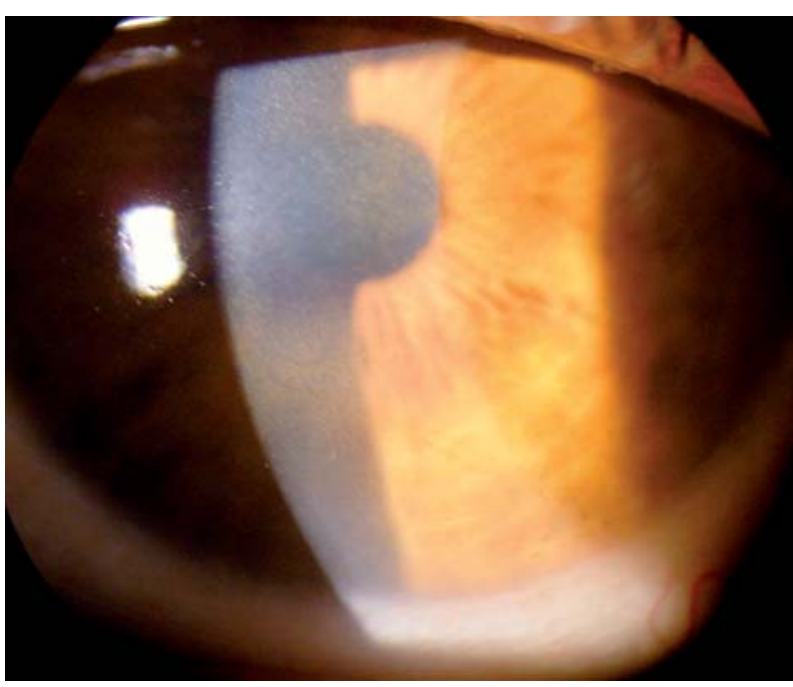

Fig. 4: Melanoqueratois estriada: discreta pigmentación corneal subepitelial difusa posterior a tratamiento de la melanosis conjuntival con mitomicina tópica.

ría del pigmento está contenido en melanoblastos situados en la capa basal del epitelio conjuntival. La córnea normal no contiene células pigmentadas. Sin embargo, en determinadas circunstancias, los melanoblastos limbares proliferan, migran hacia las capas basales del epitelio corneal, y reelaboran su pigmento. El estímulo principal de esta proliferación inusual son las agresiones corneales, especialmente si se localizan a una distancia crítica de limbo, menor a $4 \mathrm{~mm}$ (1). En personas de piel oscura con limbos marcadamente pigmentados, esta pigmentación progresa bajo el epitelio intacto, y se distribuye en la córnea formando bandas con base en limbo, y ápex dirigido hacia la lesión corneal, produciendo una opacidad local permanente o una pigmentación difusa corneal (2) que se conoce como melanoqueratosis estriada (ME). A menudo la ME se acompaña de vascularización corneal, y de despigmentación del área limbar del que parten los melanocitos (3).

En personas de raza blanca hay un caso descrito de ME, asociado a erosión corneal tras recibir ocho inyecciones de 5-fluoruracilo subconjuntival posttrabeculectomía (4). Tanto este fármaco, como la epinefrina tópica y las fenotiazinas, se han asociado a pigmentación cutánea y ocular. La invasión corneal por pigmento también puede originarse desde mela- nosis cancerosas y precancerosas conjuntivales cercanas a limbo.

Nuestra paciente es una mujer de piel clara, con dos lesiones conjuntivales pigmentadas cuya biopsia no mostró atipia o melanocitos anormales. La $\mathrm{ME}$, descrita en personas de color, se ha relacionado con agresiones corneales tan variadas como paracentesis de cámara anterior, queratoplastias, cirugía de cataratas, traumatismos y en queratitis de orígenes diversos. Los casos más severos fueron los asociados a queratoplastia penetrante o a cirugía de cataratas que cursaron con inflamación prolongada (3). En nuestro caso la ME, presente únicamente en el ojo con erosión corneal recurrente, sugiere que éste es el estímulo responsable de la migración de melanocitos limbares o conjuntivales hacia la córnea. Desconocemos si este depósito de pigmento subepitelial puede debilitar la reepitelización y por tanto predisponer a su vez a erosiones corneales, y tampoco tenemos información sobre su manejo a excepción de evitar el estímulo que promueve la ME. La mitomicina $\mathrm{C}$ en aplicación tópica ha demostrado su eficacia en el tratamiento de la melanosis adquirida primaria conjuntival (5). Aunque no se detalla su efecto en la extensión corneal de esta enfermedad, en estos casos se han apreciado alteraciones del epitelio corneal resueltas con el cese del tratamiento (5). En nuestro caso este tratamiento disminuyó la fuente de pigmentación conjuntival a pesar de que la biopsia, quizás insuficiente, no mostró atipia conjuntival. No afectó sin embargo el curso de la melanosis corneal.

\section{BIBLIOGRAFÍA}

1. Michaelson IC. Proliferation of limbal melanoblasts into the cornea in response to a corneal lesion; an experimental study. Br J Ophthalmol 1952; 36: 657-665.

2. Lemp MA. Striate melanokeratosis. Arch Ophthalmol 1991; 109: 917.

3. Cowan TH. Striate melanokeratosis in negroes. Am J Ophthalmol 1964; 57: 443-449.

4. Stank TM, Krupin T, Feitl ME. Subconjunctival 5-fluoruracil-induced transient striate melanokeratosis. Arch Ophthalmol 1990; 108: 1210.

5. Pe'er J, Frucht-Pery J. The Treatment of Primary Acquired Melanosis (PAM) With atypia by topical mitomycin C. Am J Ophthalmol 2005; 139:229-234. 
